Correspondence

Michelle L. McKechnie mmckechnie@nchecr.

unsw.edu.au

Received 14 December 2010 Accepted 17 March 2011

\section{The prevalence of urogenital micro-organisms detected by a multiplex PCR-reverse line blot assay in women attending three sexual health clinics in Sydney, Australia}

\author{
Michelle L. McKechnie, ${ }^{1,2}$ Richard J. Hillman, ${ }^{2}$ Rachel Jones, ${ }^{1,3}$ \\ Penelope C. Lowe, ${ }^{3}$ Deborah L. Couldwell, ${ }^{3}$ Stephen C. Davies, ${ }^{4}$ \\ Fiona King, ${ }^{4}$ Fanrong Kong ${ }^{2}$ and Gwendolyn L. Gilbert ${ }^{2}$
${ }^{1}$ Sexually Transmitted Infections Research Centre, University of Sydney, Marian Villa, Westmead Hospital, NSW 2145, Australia
${ }^{2}$ Centre for Infectious Diseases and Microbiology, Institute of Clinical Pathology and Medical Research, Westmead, NSW 2145, Australia
${ }^{3}$ Parramatta Sexual Health Clinic, Jeffery House, 162 Marsden Street, Parramatta, NSW 2145 , Australia
${ }^{4}$ Northern Sydney Sexual Health Service, Clinic 16, Royal North Shore Hospital, St Leonard's, NSW 2065, Australia

\begin{abstract}
This study used a previously described multiplex PCR-based reverse line blot (mPCR/RLB) assay to assess the prevalence and distribution of 14 urogenital pathogens or putative pathogens, namely Neisseria gonorrhoeae, Chlamydia trachomatis, Mycoplasma genitalium, Mycoplasma hominis, Trichomonas vaginalis, Gardnerella vaginalis, Ureaplasma parvum, Ureaplasma urealyticum, Neisseria meningitidis, Streptococcus pneumoniae, Haemophilus influenzae, herpes simplex virus types 1 and 2, and human adenovirus. First-voided urine specimens and endocervical and self-collected vaginal swabs from each of 216 women attending three sexual health clinics in Sydney, Australia, were tested and the results were compared with those of reference methods for each organism. One hundred and sixty-eight women (77.7\%) had at least one and 105 (48.6\%) had more than one target organism, most commonly G. vaginalis and Ureaplasma spp. The prevalence of each of the four known sexually transmissible pathogens was $<5 \%$. Of the 216 women, $111(51.4 \%)$ reported at least one symptom consistent with genital or urethral infection, including discharge, pain or discomfort. Only G. vaginalis was detected more frequently in women with symptoms $(P=0.05)$. The specificity of the $\mathrm{mPCR} / \mathrm{RLB}$ assay compared with that of the reference methods for each organism and for all specimen types was $100 \%$. The mean sensitivities of the mPCR/RLB assay compared with those of the reference methods for self-collected vaginal swabs, cervical swabs and first-voided urine specimens for all organisms were 99.3, 98.1 and $84.6 \%$, respectively; however, these differences were not significant. There were no differences in sensitivities between specimen types for C. trachomatis, $N$. gonorrhoeae, $T$. vaginalis and $H$. influenzae, although all were found infrequently. Overall, the mPCR/RLB platform was found to be an accurate testing platform in a sexual health clinic setting.
\end{abstract}

\section{INTRODUCTION}

Pelvic inflammatory disease, infertility, ectopic pregnancy, chronic pelvic pain, neonatal morbidity and mortality, and genital cancer have been assumed to be associated with

Abbreviations: $\mathrm{Cl}$, confidence interval; mPCR/RLB, multiplex PCRbased reverse line blot; OCP, oral contraceptive pill; SPCR, single-target PCR; STI, sexually transmitted infection. sexually transmitted infections (STIs), although in many cases the exact pathogens are unknown (Simms et al., 2003). Although STIs can cause a variety of urethral and vaginal symptoms, many occur without symptoms. For example, up to $70 \%$ of men and women with gonococcal and/or chlamydial infections are symptom-free (Stamm, 1993). This potential for undiagnosed infection has significant implications for individual and population health. Both symptomatic and asymptomatic infections can cause serious complications. 
A wide range of organisms have been implicated in symptomatic urethritis in males (Bradshaw et al., 2006; Couldwell et al., 2010; Foo et al., 2004) and various genital syndromes in women. However, additional epidemiological studies are needed to determine the significance of organisms other than the recognized genital pathogens in vaginal syndromes.

The high sensitivity of PCR allows the use of various genital specimen types, including endocervical swabs, self-collected vaginal swabs and urine samples, for simultaneous detection of genital pathogens. Some of these specimens can be collected without the need for clinical examination, thus increasing their potential usefulness in screening programmes.

We previously developed a multiplex PCR-based reverse line blot (mPCR/RLB) assay (McKechnie et al., 2009), which allows simultaneous amplification of multiple targets. PCR products are identified by hybridization with target-specific probes on a membrane, which increases the sensitivity and allows the detection and identification of 14 microbial species (and potentially up to 43 ) in up to 43 clinical specimens simultaneously.

The aims of the current study were to investigate the utility of this $\mathrm{mPCR} / \mathrm{RLB}$ assay for the simultaneous detection of 14 genital pathogens or potential pathogens in different types of genital specimens and to determine their prevalence in women attending three sexual health clinics in Sydney, Australia.

\section{METHODS}

Study population and clinical specimens. The study population comprised consecutive female patients with and without vaginal symptoms attending the Parramatta Sexual Health Clinic, Clinic 16 at the Royal North Shore Hospital and the Manly Sexual Health Clinic in Sydney, Australia. The sample size was calculated with reference to the World Health Organization guidelines for STI prevalence surveys (WHO, 2007). The prevalence of Chlamydia trachomatis is estimated to be approximately $5 \%$ in the population of women attending sexual health clinics in Australia (Vajdic et al., 2005). A sample size of 203 women would be required to measure the prevalence of $C$. trachomatis within $\pm 3.0 \%$ using a $95 \%$ confidence interval (CI) calculation. Women who were unwilling or unable to provide informed consent, were under the age of 18 , were pregnant or who had no cervix were excluded. A 'case' was defined as a woman reporting one or more of the following symptoms: vaginal discharge, vaginal irritation, dysuria, urinary frequency or pelvic pain. A 'control' was a woman with none of these specific symptoms but who presented to the clinic during the study period.

Demographic and behavioural characteristics were recorded using a questionnaire completed by a healthcare worker. Specimens tested were healthcare worker-collected endocervical swabs, first-voided urine specimens and self-collected vaginal swabs. Verbal instructions by a healthcare worker and an instructional diagram were used to aid in the collection of vaginal swabs (Rose et al., 2007), which involved inserting the swab $5 \mathrm{~cm}$ into the vagina and rotation prior to removal. All specimens were stored at $4{ }^{\circ} \mathrm{C}$ at the local laboratory and transported in batches, in a cool box, to the Centre for Infectious Diseases and Microbiology. They were then stored at $4{ }^{\circ} \mathrm{C}$ until DNA extraction, which was performed within $48 \mathrm{~h}$ of collection.
Swab sticks were placed in tubes containing $100 \mu \mathrm{l}$ lysis buffer, rotated 10-15 times and then treated as described previously for urine specimens (McKechnie et al., 2009), using a Roche COBAS Amplicor extraction kit (Roche Diagnostics Australia). Extracts were stored at $4{ }^{\circ} \mathrm{C}$ if PCR was to be performed within $24 \mathrm{~h}$ or at $-70{ }^{\circ} \mathrm{C}$ until further analysis.

Laboratory testing. Testing and validation of the mPCR/RLB assay using clinical isolates and reference strains of target organisms has been described previously (McKechnie et al., 2009). The 14 target species were: Neisseria gonorrhoeae, Chlamydia trachomatis, Mycoplasma genitalium, Mycoplasma hominis, Trichomonas vaginalis, Gardnerella vaginalis, Ureaplasma parvum, Ureaplasma urealyticum, Neisseria meningitidis, Streptococcus pneumoniae, Haemophilus influenzae, herpes simplex virus type 1 (HSV-1) and HSV-2, and adenovirus. Briefly, extracted DNA from each specimen was amplified by mPCR using biotin-labelled primers, and the amplicons were hybridized to corresponding probes fixed in rows on a labelled nylon membrane. Identification was by chemiluminescence, generated by the reaction between hybridized, biotin-labelled amplicons and streptavidin-horseradish peroxidase conjugate and the subsequent addition of substrate.

Reference methods, including culture and/or single-target PCR (sPCR), were also performed as described previously and their specificity was validated (McKechnie et al., 2009). sPCRs for nine micro-organisms were performed using the same primers as in the mPCR. The targets of the sPCRs for T. vaginalis, HSV-1 and -2, M. genitalium and human adenovirus were different from those used in the mPCR

Data analysis. All analyses were performed using sPss software, version 17.0. The demographics of cases and controls were compared using a $\chi^{2}$ or Fisher's exact test, as appropriate. All tests performed were two-sided and were considered significant for a value of $P<0.05$. Univariate associations between each target organism and demographic/behavioural factors were assessed using Pearson's $\chi^{2}$ test. Variables significantly associated with the detection of each target organism were considered for multivariate analysis. However, due to the small number of significant findings, multivariate analysis could not be performed.

\section{RESULTS}

\section{Subject characteristics}

Between July 2008 and June 2009, 1807 (1031 at the Parramatta Sexual Health Clinic and 776 at the Royal North Shore Hospital and Manly Sexual Health Clinic) women attended the sexual health clinics and a total of 216 $(12.0 \%)$ women were recruited for the study. Reasons for exclusion were not recorded. All 216 women completed the questionnaire and provided all three specimens. The ages of the 216 participants ranged from 18 to 65 years (mean 29 years, median 27 years). Four participants $(1.9 \%)$ were aged 18-19 years, 46 (21.3\%) were 20-24 years, 89 $(41.2 \%)$ were $25-29$ years, $36(16.7 \%)$ were $30-34$ years and $41(19.0 \%)$ were over 35 years of age.

\section{Demographic and behavioural characteristics}

Data on sex work, previous STI diagnoses and the sexual activities of the women are shown in Table 1. Just over half of the women reported some form of contraception use, of 
Table 1. Selected characteristics of 216 women attending sexual health clinics in relation to the detection of target organisms

$P$ values were calculated by comparing the prevalence of target organisms in women with and without different behavioural characteristics.

\begin{tabular}{|c|c|c|c|}
\hline Behavioural characteristic & Frequency $[n(\%)]$ & $\begin{array}{c}\text { One or more target } \\
\text { organisms detected }[n(\%)]\end{array}$ & $P$ value \\
\hline $\begin{array}{l}\text { Vaginal sex in the last } 4 \text { weeks with any } \\
\text { partner(s) }\end{array}$ & & & 0.072 \\
\hline No & $38(17.6)$ & $25(65.8)$ & \\
\hline Yes & $178(82.4)$ & $143(80.3)$ & \\
\hline $\begin{array}{l}\text { Condom use during vaginal sex in the last } \\
4 \text { weeks with any partner(s) }\end{array}$ & & & 0.200 \\
\hline Condom always & $48(22.2)$ & $40(83.3)$ & \\
\hline Condom sometimes & $122(56.5)$ & $97(79.5)$ & \\
\hline Not stated & $8(3.7)$ & $7(87.5)$ & \\
\hline Sex work in the last 12 months & & & 0.311 \\
\hline No & $169(78.3)$ & $134(79.3)$ & \\
\hline Yes & $47(21.7)$ & $34(72.3)$ & \\
\hline Previous STI diagnosis & & & 0.273 \\
\hline No & $123(56.9)$ & $92(74.8)$ & \\
\hline Yes & $93(43.1)$ & $76(81.7)$ & \\
\hline Contraception use & & & 0.050 \\
\hline No & $93(43.1)$ & $79(84.9)$ & \\
\hline Yes & $120(55.5)$ & $89(74.2)$ & \\
\hline Not stated & $3(1.4)$ & $0(0)$ & \\
\hline Oral contraceptive pill & & & 0.773 \\
\hline No & $143(66.2)$ & $115(80.4)$ & \\
\hline Yes & $73(33.8)$ & $53(72.6)$ & \\
\hline Condoms & & & 0.890 \\
\hline No & $185(85.6)$ & $143(77.3)$ & \\
\hline Yes & $31(14.4)$ & $25(80.6)$ & \\
\hline
\end{tabular}

which the oral contraceptive pill (OCP) was the most commonly used. No contraceptive method, individually, was significantly associated with the detection of any target organism, although the study was not powered to detect such associations. However, women who reported use of any type of contraception, including OCP, were significantly less likely to have one or more target organisms detected than those using no contraception $(89 / 120$ vs $79 / 96 ; P=0.05)$.

\section{Symptoms}

Of the 216 women enrolled in the study, 111 (51.4\%) reported one $(63,29.2 \%)$ or more $(48,22.2 \%)$ symptoms: vaginal discharge, 61 (55.0\%); vaginal itch or irritation, 57 (51.4\%); dysuria, 16 (14.4\%); urinary frequency, 12 (10.8\%) and/or pelvic pain, $25(22.5 \%)$. Eleven women $(9.9 \%)$ reported other symptoms including genital lumps, abnormal vaginal odour, cysts and abnormal vaginal bleeding.

\section{Prevalence of target organisms}

At least one target organism was detected in 168 women $(77.8 \%)$, and more than one in 105 women $(48.6 \%)$. The highest rate of detection of target species was in women aged
20-29 years $(105 / 168 ; 62.5 \%)$. One or more target species were identified in at least one specimen from 91/111 women $(82.0 \%)$ with vaginal symptoms and 77/105 asymptomatic women $(73.3 \%)$. Two or more target organisms were identified in 59 symptomatic women $(53.2 \%)$ and 46 asymptomatic women ( $43.8 \%$ ) (differences not significant). The most frequently detected species were G. vaginalis, Ureaplasma spp. and M. hominis. All recognized sexually transmissible pathogens - N. gonorrhoeae, C. trachomatis, $M$. genitalium, T. vaginalis and HSV - were identified infrequently (all $<5 \%$ ). The most commonly detected of these was C. trachomatis (3.7\%); all positive results were in women aged $<34$ years $(8 / 8 ; 100 \%)$.

Fig. 1 shows the prevalence of each micro-organism by symptom status. G. vaginalis and U. parvum had the highest prevalence rates of $50.9 \%(110 / 216)$ and $47.7 \%(103 / 216)$, respectively. G. vaginalis was detected significantly more frequently in women with symptoms than in those without $(62 / 111$ vs $48 / 105 ; P=0.05)$. There were no significant differences in detection rates between symptomatic and asymptomatic women for any other micro-organism.

Table 2 shows the prevalence of each species by age. C. trachomatis, N. gonorrhoeae, M. genitalium and U. urealyticum 


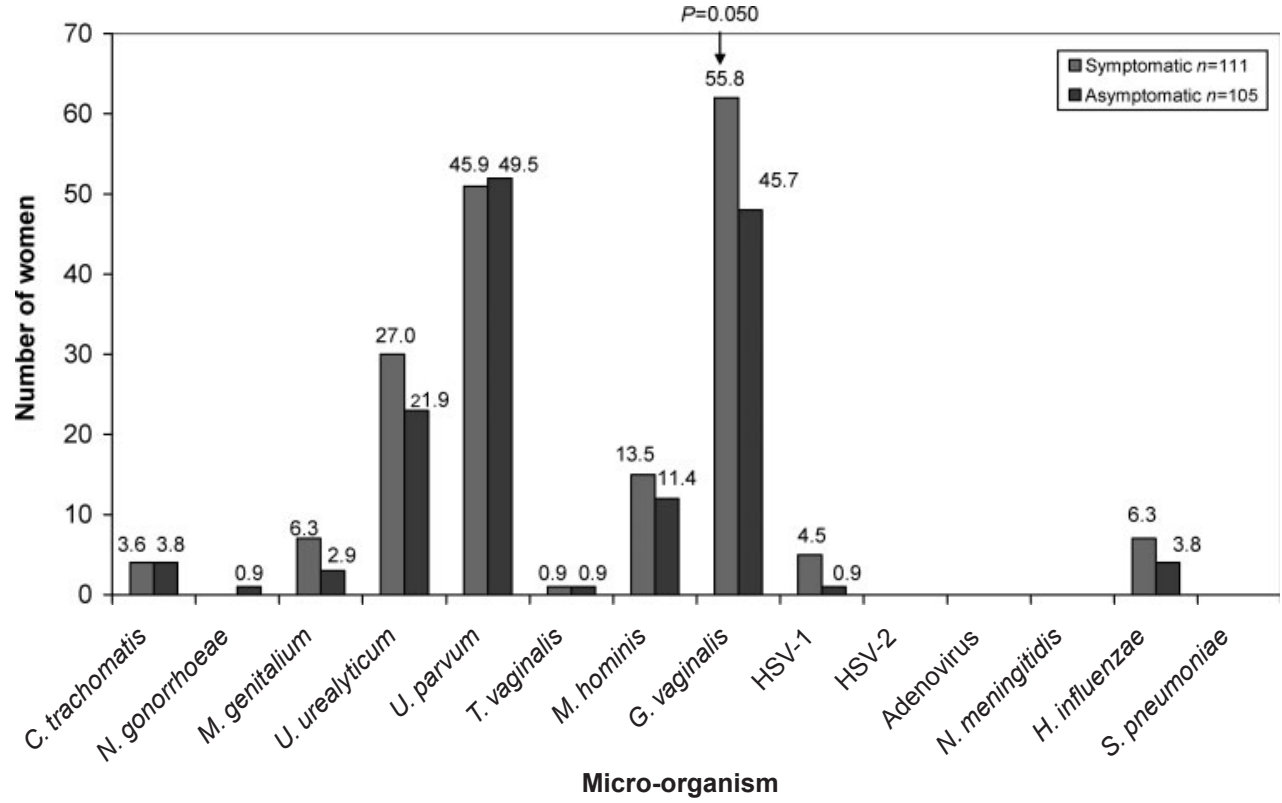

Fig. 1. Detection of 14 urogenital organisms from any specimen from women with and without vaginal symptoms. Numbers on the bars indicate the percentage of women with the specified target organism detected out of 111 symptomatic women and 105 asymptomatic women; the $y$-axis shows number of women positive for each organism. G. vaginalis was detected significantly more frequently in women with vaginal symptoms than in women without symptoms $(P<0.05)$.

were detected more frequently in women aged $<25$ years than in any other group. However, the differences were not significant.

Twenty-five of 27 women in whom $M$. hominis was detected also had $G$. vaginalis detected $(P<0.001)$ and

Table 2. Detection of 14 urogenital organisms from any specimen by age group

Multiple species were identified in some women. HSV-2, human adenovirus, $N$. meningitidis and S. pneumoniae were not detected in any specimens. There were no significant differences in the prevalence of target organisms between different age groups.

\begin{tabular}{|lcccc|}
\hline $\begin{array}{l}\text { Microbial } \\
\text { species }\end{array}$ & \multicolumn{4}{c}{ Age group (years) [n (\%)] } \\
\cline { 2 - 5 } & $\begin{array}{c}\mathbf{2 5} \\
(\boldsymbol{n}=\mathbf{5 0})\end{array}$ & $\begin{array}{c}\mathbf{2 5}-\mathbf{2 9} \\
(\boldsymbol{n}=\mathbf{8 9})\end{array}$ & $\begin{array}{c}\mathbf{3 0 - 3 4} \\
(\boldsymbol{n}=\mathbf{3 6})\end{array}$ & $\begin{array}{c}>\mathbf{3 5} \\
(\boldsymbol{n}=\mathbf{4 1})\end{array}$ \\
\hline C. trachomatis & $5(10)$ & $1(1.1)$ & $2(5.5)$ & $0(0)$ \\
N. gonorrhoeae & $1(2)$ & $0(0)$ & $0(0)$ & $0(0)$ \\
M. genitalium & $5(10)$ & $3(3.4)$ & $1(2.8)$ & $1(2.4)$ \\
U. urealyticum & $16(32)$ & $15(16.8)$ & $8(2.2)$ & $14(34.1)$ \\
U. parvum & $26(52)$ & $37(41.6)$ & $17(47.2)$ & $23(56.1)$ \\
T. vaginalis & $1(2)$ & $0(0)$ & $0(0)$ & $1(2.4)$ \\
M. hominis & $6(12)$ & $7(7.9)$ & $5(13.9)$ & $9(22.0)$ \\
G. vaginalis & $29(58)$ & $42(47.2)$ & $15(41.7)$ & $24(58.5)$ \\
HSV-1 & $2(4)$ & $1(1.1)$ & $1(2.8)$ & $2(4.9)$ \\
H. influenzae & $2(4)$ & $5(5.6)$ & $0(0)$ & $4(9.8)$ \\
\hline
\end{tabular}

over half of these women $(15 / 25 ; 60.0 \%)$ had vaginal symptoms.

\section{Specimen types and performance}

The sensitivity and specificity of the mPCR/RLB method were compared with those of reference methods for each specimen type and each target species (Table 3). Positive $\mathrm{mPCR} / \mathrm{RLB}$ results were confirmed by reference methods for all species in all specimen types, i.e. the specificity was $100 \%$. The overall sensitivities of the mPCR/RLB assay for all target organisms were 99.3, 98.1 and $84.6 \%$ for self-collected vaginal swabs, endocervical swabs and first-voided urine specimens, respectively. Endocervical swabs were the most sensitive specimen type for the detection of M. genitalium, U. urealyticum, U. parvum and $G$. vaginalis by the mPCR/RLB assay. Self-collected vaginal swabs were the most sensitive for the detection of M. hominis and HSV-1. However, none of these differences was statistically significant for individual species.

\section{DISCUSSION}

The mPCR/RLB assay detected a wide range of potential genital pathogens in healthcare worker-collected cervical swabs, self-collected vaginal swabs and first-voided urine specimens from women attending sexual health clinics in Sydney. By careful comparison of results with those of 
Table 3. Performance of the mPCR/RLB assay compared with the reference method for each organism

HSV-2, human adenovirus, N. meningitidis and S. pneumoniae were not detected in any specimens.

\begin{tabular}{|c|c|c|c|c|c|}
\hline $\begin{array}{l}\text { Target species: no. } \\
\text { positive }(\%) \text { by } \\
\text { reference method }\end{array}$ & $\begin{array}{c}\text { mPCR/RLB } \\
\text { (no. positive) }\end{array}$ & Specimen $^{*}$ & $\begin{array}{c}\text { Sensitivity } \\
{[\%(95 \% \text { CI })]}\end{array}$ & $\begin{array}{l}\text { NPV } \\
(\%) \dagger\end{array}$ & Reference method (target gene) \\
\hline C. trachomatis: 8 (3.7) & 8 & All (Cx, FVU, Vag) & $100.0( \pm 0.1670)$ & 100.0 & $\begin{array}{l}\text { Roche Cobas Amplicor PCR (cryptic } \\
\text { plasmid) and BD Viper System (plasmid) }\end{array}$ \\
\hline N. gonorrhoeae: 1 (0.5) & 1 & All (Cx, FVU, Vag) & - & - & $\begin{array}{l}\text { Roche Cobas Amplicor PCR (cryptic } \\
\text { plasmid) }\end{array}$ \\
\hline \multirow[t]{3}{*}{ M. genitalium: 10 (4.6) } & 10 & $\mathrm{Cx}$ & $100.0( \pm 0.1423)$ & 100.0 & sPCR (16S rRNA gene) \\
\hline & 9 & FVU & $90.0( \pm 0.2150)$ & 99.5 & \\
\hline & 9 & Vag & $90.0( \pm 0.2150)$ & 99.5 & \\
\hline \multirow[t]{3}{*}{ M. hominis: 27 (12.5) } & 26 & $\mathrm{Cx}$ & $96.0( \pm 0.1033)$ & 99.5 & sPCR (16S rRNA gene) \\
\hline & 24 & FVU & $88.9( \pm 0.1292)$ & 98.4 & \\
\hline & 27 & Vag & $100.0( \pm 0.0629)$ & 100.0 & \\
\hline \multirow{3}{*}{$\begin{array}{l}\text { U. urealyticum: } 53 \\
(24.5)\end{array}$} & 53 & $\mathrm{Cx}$ & $100.0( \pm 0.0339)$ & 100.0 & sPCR (multi-banded antigen gene) \\
\hline & 52 & FVU & $98.1( \pm 0.0574)$ & 99.3 & \\
\hline & 51 & Vag & $96.2( \pm 0.0659)$ & 98.8 & \\
\hline \multirow[t]{3}{*}{ U. parvum: 103 (47.7) } & 103 & $\mathrm{Cx}$ & $100.0( \pm 0.0180)$ & 100.0 & sPCR (multi-banded antigen gene) \\
\hline & 100 & FVU & $97.1( \pm 0.0397)$ & 97.4 & \\
\hline & 99 & Vag & $96.1( \pm 0.0434)$ & 96.5 & \\
\hline T. vaginalis: $2(0.9)$ & 2 & All (Cx, FVU, Vag) & - & - & sPCR (18S rRNA gene) \\
\hline \multirow[t]{3}{*}{ G. vaginalis: 110 (50.9) } & 110 & $\mathrm{Cx}$ & $100.0( \pm 0.0169)$ & 100.0 & sPCR (16S rRNA gene) \\
\hline & 105 & FVU & $95.4( \pm 0.0439)$ & 95.4 & \\
\hline & 108 & Vag & $98.2( \pm 0.0335)$ & 98.1 & \\
\hline \multirow[t]{3}{*}{ HSV-1: 6 (2.8) } & 5 & $\mathrm{Cx}$ & $83.3( \pm 0.2854)$ & 99.1 & sPCR $(p o l)$ \\
\hline & 4 & FVU & $66.7( \pm 0.3059)$ & 99.1 & \\
\hline & 6 & Vag & $100.0( \pm 0.2020)$ & 100.0 & \\
\hline H. influenzae: 11 (5.1) & 11 & All (Cx, FVU, Vag) & $100.0( \pm 0.1325)$ & 100.0 & sPCR $(g y r B)$ \\
\hline
\end{tabular}

${ }^{\star} \mathrm{Cx}$, Endocervical swab; FVU, first-voided urine; Vag, self-collected vaginal swab.

$\dagger N P V$, Negative predictive value, defined as the proportion of those with a negative test result who did not have the disease.

reference methods for individual organisms, we demonstrated that this assay had high levels of sensitivity and specificity.

Overall, 202 target organisms were detected in 91/111 symptomatic women $(82.0 \%)$ and 177 organisms in $77 /$ 105 asymptomatic women (73.0\%). Multiple organisms were identified in approximately equal numbers of women with and without symptoms. No mixed infections with $C$. trachomatis and $N$. gonorrhoeae were detected. The highest rates of detection were in the 20-29-year age group, which corresponds with the age of highest risk for STIs (de Visser et al., 2003). The most commonly detected organisms were G. vaginalis $(50.9 \%)$, U. parvum $(47.7 \%)$ and $U$. urealyticum $(25.0 \%)$ at rates comparable to those reported by others among similar populations (Fox et al., 1999; Volgmann et al., 2005; Wang, 2000).

The prevalence of C. trachomatis was $3.7 \%$; all cases were in women under the age of 34 . This is similar to that in a sample of women attending general practices in Melbourne (Hocking et al., 2006). Chlamydia was detected in similar proportions of women with and without symptoms. Of the four symptomatic women with $C$. trachomatis detected, two reported vaginal discharge and two complained of vaginal irritation and itch. However, three of these women were co-infected with other organisms.

Both M. hominis and G. vaginalis are strongly associated with bacterial vaginosis (Rosenstein et al., 1996). In our study, most women in whom $M$. hominis was found also had G. vaginalis $(P<0.001)$ and over half of them had vaginal symptoms. In contrast, $M$. hominis itself was detected equally in women with and without vaginal symptoms.

G. vaginalis was detected in a greater proportion of symptomatic patients than controls, although vaginal discharge was the only symptom that was significantly associated with it $(P=0.002)$.

The most commonly reported contraception method was the OCP, as reported in women generally in Australia (Richters et al., 2003). Only $14 \%$ of the women reported condom use, which is lower than reported for the general population (21.4\%; Richters et al., 2003). However, in response to questions about their sexual history, 48 women $(22.2 \%)$ reported using condoms during vaginal sex. This 
suggests that the reported contraceptive use may be unreliable. Close to half of all participants reported no contraceptive use. Overall, women who reported the use of any type of contraception were less likely to have a target organism detected than women using no contraception $(P=0.05)$. The reason for this is not clear, as a higher rate of infection or colonization with sexually transmissible organisms would be expected in women using non-barrier contraceptive methods such as the OCP, which was the most commonly used method.

In this study, using sPCR as the gold standard, first-voided urine specimens from women had the lowest overall sensitivity $(84.6 \%)$ when compared with endocervical $(98.1 \%)$ and self-collected $(99.3 \%)$ vaginal specimens. Using the mPCR/RLB assay and cervical swabs as the gold standard, the sensitivities of first-voided urine and selfcollected vaginal swabs were 93.5 and $95.9 \%$, respectively.

Naturally, sensitivities and specificities in different specimens varied for different organisms. For example, the $\mathrm{mPCR} / \mathrm{RLB}$ assay was more sensitive in detecting $M$. hominis and G. vaginalis in self-collected vaginal swabs than in first-voided urine from the same women, whereas it was more sensitive for first-voided urine specimens than self-collected vaginal swabs for detecting Ureaplasma spp.

Many authors have advocated the use of self-collected vaginal swabs for the detection of Chlamydia and other STIs, as they are more acceptable, convenient and effective, and increase the rate of screening when compared with endocervical swabs collected by healthcare workers (Nelson et al., 2003; Skidmore et al., 2006; Tanaka et al., 1998; Thomas et al., 1998). In addition, unlike urine specimens, self-collected vaginal swabs do not require stringent transport requirements, may be transported at room temperature to the laboratory and do not require centrifugation or incubation processing steps. Therefore, they are less susceptible to false-negative PCR results due to transport or processing issues compared with urine specimens.

Recent studies have shown that PCR testing for Chlamydia using self-collected vaginal swabs is just as sensitive as using endocervical specimens (Garrow et al., 2002; Schachter et al., 2003). Detection rates of target organisms using self-collected vaginal swabs in this study were similar to those for endocervical specimens. For M. hominis and HSV-1 detection, vaginal swabs were more sensitive than urine or endocervical specimens.

This study had a number of limitations. It was based on a convenience sample of women attending three sexual health clinics in Sydney who were classified as 'cases' and 'controls' only on the basis of self-reported symptoms. However, the main aim of the study was to assess the utility of the mPCR/RLB method to identify a fairly large number of putative genital pathogens simultaneously. Their prevalence in this population and the relationships of individual organisms to selected clinical and demographic characteristics were also assessed. Interpretation of the clinical significance of these pathogens in individual women would require more detailed clinical and microbiological data, especially microscopy of wet preparations and Gram stains of genital smears.

We showed that the mPCR/RLB assay is sensitive and specific for detection of a wide range of organisms in all types of specimen used in this study. Different sexual networks were analysed including a small proportion of samples from sex workers. The poor correlation between the detection of these organisms and behavioural characteristics typically associated with sexual transmission could suggest that other factors may be involved in their acquisition. Many of these organisms have been implicated in the development of non-gonococcal urethritis in males. In women, the potential pathological effects of infection with C. trachomatis, $N$. gonorrhoeae, T. vaginalis, HSV, S. pneumoniae and recently M. genitalium (Jensen et al., 2004; Manhart et al., 2007), and the association of G. vaginalis in bacterial vaginosis, have been established. However, the pathogenic potential of $U$. urealyticum, $U$. parvum, $H$. influenzae, $N$. meningitidis and human adenovirus in the female genital tract remains the subject of ongoing research. It is hoped that the results of this study will add to the body of evidence published to date on the epidemiology of these microorganisms in these anatomical sites.

The $\mathrm{mPCR} / \mathrm{RLB}$ assay is a user-friendly testing platform suitable for use in sexual health clinics and possibly in community settings. It is possible to investigate the presence of a wide range of potential pathogens in women who decline a physical examination. Further investigations using this technology may allow a greater understanding of the roles of other organisms in the development of conditions such as acute pelvic inflammatory disease, in which the pathogens are frequently not identified using current testing methods.

\section{ACKNOWLEDGEMENTS}

We acknowledge the contributions made from clinical research teams at the Parramatta Sexual Health Clinic, Northern Sydney Sexual Health Service, Centre for Infectious Diseases and Microbiology (CIDM) Laboratory Services and Sexually Transmitted Infections Research Centre and all the women who participated in the study. The research was supported by a grant from CIDM Public Health.

\section{REFERENCES}

Bradshaw, C. S., Tabrizi, S. N., Read, T. R., Garland, S. M., Hopkins, C. A., Moss, L. M. \& Fairley, C. K. (2006). Etiologies of nongonococcal urethritis: bacteria, viruses, and the association with orogenital exposure. J Infect Dis 193, 336-345.

Couldwell, D. L., Gidding, H. F., Freedman, E. V., McKechnie, M. L., Biggs, K., Sintchenko, V. \& Gilbert, G. L. (2010). Ureaplasma urealyticum is significantly associated with non-gonococcal urethritis in heterosexual Sydney men. Int J STD AIDS 21, 337-341.

de Visser, R. O., Smith, A. M., Rissel, C. E., Richters, J. \& Grulich, A. E. (2003). Sex in Australia: heterosexual experience and recent 
heterosexual encounters among a representative sample of adults. Aust N Z J Public Health 27, 146-154.

Foo, C., Browne, R. \& Boag, F. (2004). Retrospective review of the correlation of symptoms, signs and microscopy with the diagnosis of Chlamydia trachomatis in men. Int J STD AIDS 15, 319-321.

Fox, K. K., Isbey, S. F. \& Cohen, M. S. (1999). Urethritis, epidydimitis, orchitis, prostatitis. In Clinical Infectious Diseases: a Practical Approach, pp. 669-679. Edited by R. K. Root, L. Corey \& W. E. Stamm. New York: Oxford University Press.

Garrow, S. C., Smith, D. W. \& Harnett, G. B. (2002). The diagnosis of chlamydia, gonorrhoea, and trichomonas infections by self obtained low vaginal swabs, in remote northern Australian clinical practice. Sex Transm Infect 78, 278-281.

Hocking, J. S., Lim, M. S., Vidanapathirana, J., Read, T. R. \& Hellard, M. (2006). Chlamydia testing in general practice - a survey of Victorian general practitioners. Sex Health 3, 241-244.

Jensen, J. S., Björnelius, E., Dohn, B. \& Lidbrink, P. (2004). Comparison of first void urine and urogenital swab specimens for detection of Mycoplasma genitalium and Chlamydia trachomatis by polymerase chain reaction in patients attending a sexually transmitted disease clinic. Sex Transm Dis 31, 499-507.

Manhart, L. E., Holmes, K. K., Hughes, J. P., Houston, L. S. \& Totten, P. A. (2007). Mycoplasma genitalium among young adults in the United States: an emerging sexually transmitted infection. Am J Public Health 97, 1118-1125.

McKechnie, M. L., Hillman, R., Couldwell, D., Kong, F., Freedman, E., Wang, H. \& Gilbert, G. L. (2009). Simultaneous identification of 14 genital microorganisms in urine by use of a multiplex PCR-based reverse line blot assay. J Clin Microbiol 47, 1871-1877.

Nelson, D. B., Bellamy, S., Gray, T. S. \& Nachamkin, I. (2003). Selfcollected versus provider-collected vaginal swabs for the diagnosis of bacterial vaginosis: an assessment of validity and reliability. J Clin Epidemiol 56, 862-866.

Richters, J., Grulich, A. E., de Visser, R. O., Smith, A. M. \& Rissel, C. E. (2003). Sex in Australia: contraceptive practices among a representative sample of women. Aust N Z J Public Health 27, 210-216.

Rose, S. B., Lawton, B. A., Bromhead, C., MacDonald, E. J. \& Lund, K. A. (2007). Self-obtained vaginal swabs for PCR chlamydia testing: a practical alternative. Aust N Z J Obstet Gynaecol 47, 415-418.
Rosenstein, I. J., Morgan, D. J., Sheehan, M., Lamont, R. F. \& TaylorRobinson, D. (1996). Bacterial vaginosis in pregnancy: distribution of bacterial species in different Gram-stain categories of the vaginal flora. J Med Microbiol 45, 120-126.

Schachter, J., McCormack, W. M., Chernesky, M. A., Martin, D. H., Van Der Pol, B., Rice, P. A., Hook, E. W., III, Stamm, W. E., Quinn, T. C. \& Chow, J. M. (2003). Vaginal swabs are appropriate specimens for diagnosis of genital tract infection with Chlamydia trachomatis. J Clin Microbiol 41, 3784-3789.

Simms, I., Warburton, F. \& Weström, L. (2003). Diagnosis of pelvic inflammatory disease: time for a rethink. Sex Transm Infect 79, 491494.

Skidmore, S., Horner, P., Herring, A., Sell, J., Paul, I., Thomas, J., Caul, E. O., Egger, M., McCarthy, A. \& other authors (2006). Vulvovaginal-swab or first-catch urine specimen to detect Chlamydia trachomatis in women in a community setting? J Clin Microbiol 44, 4389-4394.

Stamm, W. E. (1993). Toward control of sexually transmitted chlamydial infections. Ann Intern Med 119, 432-434.

Tanaka, M., Nakayama, H., Yoshida, H., Takahashi, K., Nagafuji, T., Hagiwara, T. \& Kumazawa, J. (1998). Detection of Chlamydia trachomatis in vaginal specimens from female commercial sex workers using a new improved enzyme immunoassay. Sex Transm Infect 74, 435-438.

Thomas, B. J., Pierpoint, T., Taylor-Robinson, D. \& Renton, A. M. (1998). Sensitivity of the ligase chain reaction assay for detecting Chlamydia trachomatis in vaginal swabs from women who are infected at other sites. Sex Transm Infect 74, 140-141.

Vajdic, C. M., Middleton, M., Bowden, F. J., Fairley, C. K. \& Kaldor, J. M. (2005). The prevalence of genital Chlamydia trachomatis in Australia 1997-2004: a systematic review. Sex Health 2, 169-183.

Volgmann, T., Ohlinger, R. \& Panzig, B. (2005). Ureaplasma urealyticum - harmless commensal or underestimated enemy of human reproduction? A review. Arch Gynecol Obstet 273, 133-139.

Wang, J. (2000). Bacterial vaginosis. Prim Care Update Ob Gyns 7, $181-185$.

WHO (2007). Fact Sheet No 110: Sexually Transmitted Infections. Geneva: World Health Organization. 